\title{
Gestión para el desarrollo de vivienda social. Los proyectos de viviendas 100\% subsidiadas Plaza de la Hoja y Villas de San Pablo en Colombia
}

\author{
Angela Bedoya-Ruiz. Universidad de La Salle, Bogotá, Colombia. \\ Carlos Agudelo-Rodríguez. Universidad de La Salle, Bogotá, Colombia. \\ Helmuth Ramos-Calonge. Universidad de La Salle, Bogotá, Colombia. \\ Clara Juliao-Vargas. Corporación Universitaria Minuto de Dios, Bogotá, Colombia.
}

RESUMEN | Un aspecto clave en los procesos de integración urbana de hogares socioeconómicamente vulnerables es el acceso a la vivienda social. Sin embargo, las políticas y programas diseñados para su provisión se encuentran en una posición ambigua entre las políticas sociales de los Estados, al perseguir simultáneamente objetivos sociales respecto de la población en situación de pobreza, y crecimiento económico mediante la dinamización del sector construcción. Esto contribuye a su desarticulación con los procesos de planificación territorial, que generalmente requieren de visión de largo plazo. Este artículo reflexiona sobre el rol de la gestión y planificación en la provisión de vivienda social, con miras a la definición de variables que puedan considerarse para la evaluación de proyectos. Se concluye, a partir del estudio de dos casos del Programa de Vivienda Gratuita, que la gestión debe abordarse de manera integral, involucrando acciones de coordinación interinstitucional, instrumentos de planificación territorial y actividades para el seguimiento de los hogares beneficiados.

PALABRAS CLAVE | gestión urbana, política habitacional, vivienda.

ABSTRACT | Social housing is conceived as a critical element in the urban integration processes of either social or economically vulnerable homes. However, the policies and programs for social housing provision stand in an ambiguous position because they simultaneously aim for social goals and strive for economic growth by promoting the construction sector. The latter contributes to the disconnection between its provision and processes of spatial planning that usually require a long-term vision. The following article reflects on planning and its role in social housing provision, toward the definition of variables that could be considered for different projects. Two Colombian social housing projects of the "Vivienda Gratuita" housing program are analyzed to study these issues. The study concludes that social housing management must be conceived integrally, simultaneously involving actions in three fronts: interinstitutional and multilevel coordination, planning and land management instruments, and monitoring of benefited households.

KEYWORDs | urban management, housing policy, housing.

Recibido el 12 de julio de 2019, aprobado el 6 de diciembre de 2019.

E-mails: A. Bedoya-Ruiz, ambedoya@unisalle.edu.co |C. Agudelo-Rodríguez, cagudelo@lasalle.edu.co | H. Ramos-Calonge, hramos@ unisalle.edu.co | C. Juliao-Vargas, cjuliao@uniminuto.edu 


\section{Introducción}

La provisión de vivienda social se concibe como una medida importante en el contexto de aquellas conducentes a mejorar las condiciones de integración urbana, entre las cuales se cuentan las de inclusión social de hogares socioeconómicamente vulnerables. Sin embargo, si bien su relevancia es indiscutible a la hora de pensar en escenarios de igualdad en el ámbito urbano, los programas creados para la provisión de vivienda social difícilmente son considerados parte de la política social de los países. A menudo ocupan un lugar ambiguo en los sistemas de bienestar de los Estados, aunque una vez ejecutados son incluidos como ayudas en esta materia. Ello se debe -en parte- a que, en coyunturas de estabilización y crecimiento económico, la política habitacional también es vista como una oportunidad para el establecimiento de estímulos que favorecen tanto la actividad urbanizadora como al sector de la construcción, este último considerado clave para la formación de capital y generación de empleo (Pareja-Eastway \& Sánchez-Martínez, 2012).

Por vivienda social nos referimos a aquella que se encuentra bajo algún tipo de protección o intervención estatal, lo cual posibilita su acceso a hogares que no están en capacidad para hacerlo en condiciones normales de mercado (CamachoCardona, 1998; Leal-Maldonado, 2005; Scanlon et al., 2014). No obstante, vale la pena aclarar que no existe un concepto de vivienda social aplicable o aceptado de manera universal, ya que en los distintos países existen diferencias en lo que se refiere - por ejemplo- al carácter de los agentes que la promueven, esquemas de tenencia que facilitan su acceso, grado de intervención pública y prioridad otorgada a la política habitacional, entre otros (Pareja-Eastway \& Sánchez-Martínez, 2012). De cualquier manera, los estudios comparados de políticas habitacionales siguen siendo pertinentes y revelan algunas similitudes en lo que respecta a su implementación y al desarrollo de vivienda social durante las últimas tres décadas en diferentes países y regiones.

En concordancia con lo anterior, actualmente se reconocen diversos efectos que dan cuenta de la contracción de la escala del apoyo y del capital estatal, tanto financiero como organizacional y humano, requerido para mostrar resultados en la provisión de vivienda social. En esta línea, la intervención del Estado ha ido migrando hacia medidas para aumentar los ingresos de los hogares desde la demanda y con modelos administrativos más fragmentados (Cuervo \& Jaramillo, 2010; Maclennan, 2005). Se trata de un proceso correlacionado con otras circunstancias, como el aumento de esquemas de tenencia en propiedad para garantizar el acceso a la vivienda y disminución de unidades de carácter público para alquiler; la aproximación a la oferta desde un enfoque cuantitativo, que tiene repercusiones en la disminución de la calidad de su materialización y del ambiente urbano donde se localiza; y una preferencia por el desarrollo de vivienda nueva, esto último ligado a la ya mencionada apuesta simultánea por objetivos sociales, y fines fiscales y de estabilización económica (Arango, 2001; Kleinman, 1996; Sugranyes, 2006).

Los aspectos mencionados han llevado a concluir que las recientes políticas habitacionales han tenido un impacto limitado, así como efectos regresivos, especialmente en el alcance de logros sociales y medioambientales (Maclennan, 2005; 
ONU-Hábitat \& Banco de Desarrollo de América Latina [CAF], 2014). En regiones como la latinoamericana, donde la vivienda social se produce de manera masiva, la ejecución de dichas políticas todavía muestra rezagos frente a las necesidades habitacionales de la población, y ello inicialmente en términos cuantitativos, pero de manera muy importante en la actualidad en términos cualitativos, dada la baja calidad de la oferta de vivienda ya desarrollada (Rodríguez \& Sugranyes, 2005). Además, el hecho de que su ejecución y diseño sean especialmente sensibles a los devenires políticos y modelos económicos de los países, contribuye a su desarticulación con los procesos de planificación espacial en ámbitos locales, que usualmente requieren de una visión de largo plazo y la confluencia de diversos proyectos sectoriales para el logro de ambientes urbanos y entornos metropolitanos de calidad.

El presente artículo busca, a través del estudio de dos proyectos pertenecientes al programa del gobierno colombiano de viviendas 100\% subsidiadas, señalar aspectos pertinentes para la gestión integral de la vivienda social que atañen tanto a los niveles nacionales, como a aquellas jurisdicciones donde se localizan los proyectos mismos. Lo anterior con miras a definir aspectos y variables que puedan ser tenidos en cuenta para el diseño y evaluación de los programas que se encargan de su provisión.

La primera parte del trabajo discute el rol de la planificación y gestión en la provisión de vivienda social, evidenciando su relevancia en los procesos de integración social en el ámbito urbano, fin que debería perseguirse con el desarrollo de este tipo de vivienda. Posteriormente, se relacionan los dos proyectos analizados y aspectos pertinentes de su gestión que han tenido efecto tanto en su desarrollo como en la percepción de satisfacción de los usuarios. Por último, el artículo propone algunas recomendaciones para la gestión del programa y casos estudiados. Se concluye que la gestión de la vivienda social, y de los programas para su provisión, deben abordarse de manera más amplia, que involucre tanto acciones de coordinación entre las instituciones y los múltiples agentes involucrados en su desarrollo, como el uso de instrumentos de planificación territorial y actividades para el seguimiento de los hogares beneficiados, con miras a facilitar su proceso de integración al ámbito urbano.

\section{La gestión y planificación urbana en la provisión de vivienda social}

Teniendo en cuenta que los programas para la provisión de vivienda social remiten ineludiblemente (una vez que la vivienda es materializada) a procesos sociales y comunitarios de los hogares que benefician, se considera que su diseńo y evaluación también deben ser abordados desde perspectivas y conceptos propios de los programas en ámbitos sociales. Esto implica superar la perspectiva macro, que en la actualidad se enfoca mayoritariamente en el desarrollo cuantitativo de soluciones y productos habitacionales; y asegurar que, desde su diseńo, se propenda a la coherencia entre efectos e impactos deseables, y proyectos y objetivos iniciales de los programas (Bedoya et al., 2018; Sugranyes, 2006). Para esto, todos sus componentes, así como la conducta de los actores y organizaciones externas vinculadas, deben orientarse al cumplimiento de dichos objetivos (Cohen \& Franco, 2005).

En concordancia con lo anterior, tanto para el diseño como para la evaluación de sus arreglos normativos, es necesario abarcar dos dimensiones que se encuentran de 
manera simultánea en los programas sociales: por un lado, el nivel de complejidad intermedio de estos, que los sitúa entre las políticas y los proyectos concretos que cobijan; por el otro, su condición de "contextualidad", referida a las particularidades de implementación del programa en diversos contextos, bien sean ámbitos territoriales o niveles de gobierno, cada uno con competencias y capacidades (e incluso limitaciones) específicas para la gestión del programa y sus componentes (Fernández-Ballesteros, 2011).

En el caso específico de la vivienda social, lo anterior implica que los programas encargados de su provisión deben contemplar, para su organización y gestión, el diseńo y seguimiento de un vasto rango de acciones. En un nivel superior, aquellas pertenecientes a la definición de mecanismos para los procesos de gobernanza y de coordinación multinivel e interinstitucional requeridos para el funcionamiento de políticas (en este caso, la habitacional), los programas que comprenden, y su articulación con otros de corte social. En un nivel inferior, aquellas necesarias para la inserción y materialización de la vivienda como elemento construido en contextos locales y regionales, pertenecientes al planeamiento y la gestión territorial (Bedoya et al., 2018).

Los procesos de coordinación entre diversos niveles de gobierno, instituciones y agentes involucrados en la promoción y desarrollo de la vivienda (entre los que también se encuentran la sociedad civil y agentes privados), contribuyen al logro de una adecuada inserción de la vivienda social en las escalas metropolitanas y urbanas. Esto enmarcado en políticas habitacionales que incorporen los costos asociados al emplazamiento de la vivienda y los efectos de su localización en la productividad de estos ámbitos territoriales, y no supediten su ejecución a los beneficios macroeconómicos que se puedan extraer de su desarrollo en el corto plazo (Maclennan, 2005). Para esto, se requiere que en el marco de la coordinación se invierta la posición de superioridad que generalmente adoptan los gobiernos nacionales ante las jurisdicciones más pequeñas, para empoderarlas mejorando sus capacidades de ejecución de abajo hacia arriba. Lo anterior es clave si se tiene en cuenta que son los ámbitos locales los que pueden poner en valor la gestión urbana-territorial, promover programas de acceso a servicios públicos de alcance local, desarrollar dotacionales (CEPAL, 2003) y articular los proyectos de vivienda a los sistemas estructurantes de las ciudades.

En el nivel inferior, en lo que respecta a la materialización de la vivienda en ámbitos metropolitanos y urbanos, también se considera fundamental que los programas de provisión se articulen a las disposiciones del planeamiento territorial y a los instrumentos dispuestos para la gestión urbana (Maclennan, 2005; TorresTovar, 2008). Estos últimos contribuyen a la captura de aumentos de valor derivados de la acción urbanística y consecución de suelo tanto para la vivienda como para el desarrollo de servicios complementarios; en especial, aquellos de carácter colectivo y elementos pertenecientes a la estructura ecológica de las ciudades (Morales-Schechinger, 2005). La articulación de disposiciones de planificación territorial y programas de provisión de vivienda, tendrá finalmente efectos en la calidad habitacional de los proyectos desarrollados, considerando que la vivienda se encuentra inmersa en una serie de relaciones y servicios urbanos, el acceso a los 
cuales puede influir, en mayor o menor medida, en las condiciones de integración social de los hogares al ámbito urbano.

Asimismo, entre los propósitos de las políticas habitacionales, programas de provisión y desarrollo de los proyectos de vivienda social, debería figurar el fomento de las condiciones necesarias para facilitar la integración social de la población que buscan beneficiar (Brain et al., 2007). Esto último no es nada fácil, empezando por una definición absoluta de dichas condiciones. La integración social parte de la delimitación conceptual y estudio de la segregación social del espacio urbano como fenómeno en diversos y disímiles entornos geográficos (ejemplos en Marcuse, 2005; Musterd, 2005; Sabatini et al., 2001). Marcuse (2005) la define como la mezcla de grupos poblacionales en el ámbito urbano con relaciones fluidas y no jerarquizadas. No obstante, al igual que en la segregación, en la integración social se conjugan aspectos sociales y condiciones físicas de manera compleja y dinámica, que dificultan su delimitación.

Musterd (2003, 2005), por ejemplo, se aproxima a la integración a través de algunos aspectos que pueden incidir en ella: la configuración de los Estados de bienestar de los países, el acceso al mercado laboral y a programas educativos, el acceso a redes sociales, y características personales de los individuos. Kesteloot et al. (2006), por su parte, definen tres esferas de la integración: participación en el mercado laboral, relación entre el individuo y las instituciones del Estado de bienestar, y la posición del individuo en las redes sociales, esferas a las que se accede respectivamente por condiciones de utilidad social, de ciudadanía y de afiliación. Las tres pueden ser relacionadas con atributos socioespaciales y escalas territoriales diferenciadas — ciudad, barrio e inmediaciones de la vivienda-, a partir de los cuales podrían diseñarse mecanismos de gestión ligados a los programas de vivienda social.

Para estos autores, la integración podría pensarse como un todo a través de: i) el acceso a bienes y servicios sujetos a normas del mercado en la escala de la ciudad, aspecto que remite ineludiblemente a las condiciones relacionales determinadas por la localización de la vivienda en términos de distancia y costos implicados en el acceso a dichos servicios (como, por ejemplo, los costos asociados al transporte); ii) las condiciones de acceso a bienes colectivos y públicos, equipamientos sociales y culturales en el ámbito barrial; y iii) una disposición adecuada del espacio público y del ámbito comunal privado, que permita el fortalecimiento de redes de solidaridad y reciprocidad en el tiempo.

Sin embargo, tanto Musterd (2003) como Kesteloot et al. (2006) reconocen una limitada incidencia a la dimensión física de la integración, ya que en esta influyen también aspectos como el nivel educativo, competencias lingüísticas, o incluso sociales, que habilitan a los grupos sociales para acceder a recursos a través del mercado. El fomento de estas habilidades depende también del acceso a otros programas en materia social ligados de manera directa a los programas habitacionales.

Finalmente, y relacionado con lo anterior, una concepción amplia de la gestión debe incluir también su articulación con acciones encaminadas al empoderamiento y seguimiento de los hogares beneficiados, ligadas a los retos que conlleva la integración social. En especial, en lo que respecta al desarrollo de capacidades para acceder a fuentes de información laboral y puestos de trabajo. 


\section{Caso de estudio: dos proyectos de vivienda $\mathbf{1 0 0 \%}$ subsidiada en Colombia}

El programa de las viviendas $100 \%$ subsidiadas, también conocido como Programa de Vivienda Gratuita (en adelante PVG), fue creado en 2012 con el propósito de atender a la población urbana más vulnerable socioeconómicamente, incluyendo a hogares en condiciones "de vulnerabilidad priorizada, sin capacidad de ahorro ni acceso a crédito" (Ministerio de Vivienda, Ciudad y Territorio, 2014a, p. 60), y a familias víctimas del conflicto armado colombiano. En su primera etapa, se estableció como metas la ejecución de 100.000 unidades de vivienda de interés prioritario $^{1}$ a lo largo del país, y su adjudicación a través de una nueva modalidad de subsidio en especie que otorga el $100 \%$ de las unidades en gratuidad.

El programa se desarrolló rápidamente. Tres años después, se tenía como balance de su implementación: 100.000 unidades de viviendas construidas, 205 municipios y 29 departamentos beneficiados, y 283 proyectos (Ministerio de Vivienda, Ciudad y Territorio, 2015a). Vale la pena resaltar que la oferta habitacional desarrollada en el marco del programa se concentró en ciudades principales del país, aunque buscaba beneficiar inicialmente a ciudades intermedias y municipios pequeños. Aproximadamente el 30\% del total de las viviendas se construyó en Bogotá, Medellín, Cali, Barranquilla, Bucaramanga, Cartagena y Cúcuta, cifra que asciende al 59\% cuando se consideran todas las ciudades capitales de los departamentos colombianos.

El diseño del programa contempló la reglamentación del subsidio en especie como principal instrumento para la transferencia de las viviendas; las condiciones para su adjudicación a los hogares beneficiados; las responsabilidades otorgadas a los entes y agentes involucrados (distintos niveles de gobierno, agentes privados participantes y hogares beneficiados); y la reglamentación del esquema financiero y funcionamiento de las diferentes modalidades de gestión para el desarrollo de los proyectos. Para su financiación, se facultó al Fondo Nacional de la Vivienda, instancia del gobierno central, para la administración de los recursos del programa, a través de la constitución de una fiducia mercantil encargada de los procesos de selección de proyectos o de constructores (Ministerio de Vivienda, Ciudad y Territorio, 2015b). En concreto se encuentran tres modalidades de desarrollo:

- Privada: Compra de viviendas a promotores privados, por parte del gobierno nacional, en proyectos ya iniciados o terminados. Se efectúa por el máximo valor de la vivienda de interés prioritario (70 salarios mínimos).

- Pública: Selección de constructores para el desarrollo de proyectos de vivienda en suelos aportados a título gratuito por entidades del sector público. Se celebran patrimonios autónomos derivados y se asume un precio de compra de la vivienda inferior a 70 salarios mínimos, teniendo en cuenta el aporte previo del suelo.

1 En Colombia hay dos tipos de vivienda social, que se distinguen por su precio: la Vivienda de Interés Social (VIS), de hasta 135 salarios mínimos legales vigentes, y la Vivienda de Interés Prioritario (VIP), de hasta 70, reglamentados por el Sistema Nacional de Vivienda de Interés Social (Ley 3/1991). 
- De convenio: Adquisición de proyectos de vivienda promovidos, gestionados o construidos por entidades de los municipios o departamentos, cuando estas aporten un porcentaje de su valor. Se celebran convenios entre diferentes instituciones, dependiendo el caso. Teniendo en cuenta el aporte de un porcentaje del valor de los proyectos, el precio por el cual se compra las viviendas es inferior a los 70 salarios mínimos.

También, cuatro años después de la creación del programa, se reglamentó el Sistema Nacional de Acompañamiento e Infraestructura Social, que define responsabilidades y procedimientos para el desarrollo de la infraestructura social de los proyectos - equipamientos y servicios complementarios - y para otras actividades de seguimiento y acompañamiento social de los hogares beneficiados. No obstante, su reglamentación se dio cuando la mayoría de los proyectos ya estaban desarrollados o en proceso de desarrollo.

En este trabajo se analizan dos proyectos de Vivienda Gratuita que dan cuenta de las condiciones en las modalidades de convenio y privada: Plaza de la Hoja (Bogotá) y Villas de San Pablo (Barranquilla), respectivamente. Con la evaluación se busca dar cuenta de su gestión, delimitada por: i) los mecanismos de coordinación existentes entre entidades y niveles de gobierno y otros agentes, como sociedad civil y privados; su efectividad para el desarrollo de la vivienda, y su articulación con la oferta de otros programas sectoriales y sociales; ii) existencia de acciones e instrumentos para la promoción del acceso al suelo urbano, y la articulación del desarrollo de la vivienda con la implementación de instrumentos de planificación territorial que deriven en mejores condiciones de implementación urbana y metropolitana; y iii) fomento de condiciones que permitan el empoderamiento y participación de la población beneficiada, con miras a "asegurar" la apropiación de la vivienda recibida y su inclusión en las dinámicas económicas y sociales de la sociedad.

El análisis señalado se realiza, por un lado, a través del examen del proceso de construcción de los proyectos e identificación de aspectos relevantes de su estado de desarrollo, en el momento del estudio; por el otro, revisando la percepción de diversos agentes involucrados respecto tanto de los procesos de gestión como de la satisfacción con los resultados obtenidos (vivienda y entorno urbano recibidos).

De acuerdo con lo anterior, se utilizaron como instrumentos de análisis:

- Referentes al proceso de desarrollo de los proyectos: análisis de fuentes secundarias y documentos oficiales del programa, junto con entrevistas a agentes clave encargados o involucrados en su ejecución, pertenecientes a entidades públicas o privadas. En este respecto será de importancia contrastar el discurso de los agentes institucionales y representantes de entidades, con los arreglos normativos y definición de competencias en el marco del PVG.

- Estado de desarrollo de los proyectos: observación directa de las agrupaciones de vivienda y su entorno inmediato (aproximadamente a 300 metros a la redonda), y registro de datos en fichas de levantamiento. Del entorno donde se localizan los proyectos, fue importante identificar la existencia de servicios complementarios a la vivienda accesibles para sus residentes. En el ámbito de la agrupación de las viviendas, se registró información acerca de la existencia y estado de servicios 
complementarios, infraestructura social, espacios públicos y zonas comunales, entre otros.

- Percepción de satisfacción de la población beneficiada: se recurrió a la realización de encuestas a la población residente, junto con talleres focales realizados con residentes reconocidos como líderes por parte de la comunidad. Estas actividades permitieron conocer la perspectiva de los residentes en lo que respecta a los procesos, avances y situaciones presentadas a partir de la adjudicación de su vivienda (Tabla 1 ).

TABLA I Actividades desarrolladas en campo

\begin{tabular}{|c|c|c|}
\hline ACTIVIDAD & $\begin{array}{l}\text { PLAZA DE LA HOJA, } \\
\text { AGOSTO 6-8/20I6 }\end{array}$ & $\begin{array}{c}\text { VILLAS DE SAN PABLO, } \\
\text { JULIO 6-9/20I6 }\end{array}$ \\
\hline Grupo focal & 1 (10 participantes) & 3 (25 participantes) \\
\hline Encuesta hogares & 66 & 53 \\
\hline Entrevista líderes comunales & 1 & 1 \\
\hline \multirow{2}{*}{$\begin{array}{l}\text { Entrevista agentes institucionales / } \\
\text { privados involucrados en la imple- } \\
\text { mentación del programa }\end{array}$} & $\begin{array}{l}2 \text { (funcionarios } \\
\text { Metrovivienda) }\end{array}$ & $\begin{array}{l}4 \text { (funcionarios Secretaría de } \\
\text { Hábitat y Fundación Mario } \\
\text { Santo Domingo) }\end{array}$ \\
\hline & \multicolumn{2}{|c|}{1 (funcionarios Departamento Prosperidad Social) } \\
\hline Levantamientos datos de agrupación & 1 & 1 \\
\hline $\begin{array}{l}\text { Levantamientos datos de entorno } \\
\text { inmediato }\end{array}$ & 1 & 1 \\
\hline
\end{tabular}

FUENTE: ELABORACIÓN PROPIA

A continuación, los aspectos más relevantes del análisis realizado a los dos proyectos.

\section{Plaza de la Hoja (Bogotá)}

Proyecto desarrollado por la modalidad de convenio en el periodo 2014-2015, Plaza de la Hoja se ubica en la localidad de Puente Aranda, en Bogotá, en un sector de la ciudad ya consolidado y de carácter predominantemente industrial. Se construyó en un predio de titularidad pública que previamente pertenecía al Instituto de Desarrollo Urbano (IDU), que funcionaba como espacio de parqueo para los buses del sistema masivo de transporte urbano y por donde pasa una de sus líneas. El proyecto cuenta hoy con un total de 457 unidades de vivienda (apartamentos) distribuidos en cinco torres con alturas variables entre 10 y 15 pisos, conectadas por edificaciones de cinco pisos de manera, que su disposición conforma un espacio central.

Una particularidad de este proyecto es que el gobierno de la ciudad articuló el PVG, de iniciativa nacional, con una estrategia municipal de revitalización y desarrollo de vivienda social en áreas centrales de la ciudad, denominada Plan Urbano del Centro Ampliado de Bogotá. Dicho plan delimitó una zona (aproximadamente 25\% del área urbana de Bogotá), con base en su condición de proximidad a redes de transporte público y sectores de mayor concentración de lugares de empleo, que contaba simultáneamente con índices de ocupación inferiores al promedio de la ciudad y baja densidad poblacional (Alcaldía Mayor de Bogotá, Secretaría de 
Planeación \& Secretaría de Hábitat, 2014). Para este centro extendido de la ciudad, el plan proponía el desarrollo de proyectos residenciales destinados a hogares de diferentes ingresos, cuyo propósito era promover la mezcla social y equilibrar la concentración periférica de la vivienda de interés social y prioritaria, situada especialmente en la parte suroccidental de la capital.

Plaza de la Hoja (Figura 1), entonces, fue asumido como un proyecto piloto y a la vez emblemático de la administración municipal del momento, que buscaba demostrar que era posible el desarrollo de vivienda gratuita de interés prioritario en un suelo privilegiado de la ciudad.

\section{Figura I | Localización de Plaza de la Hoja en Bogotá}

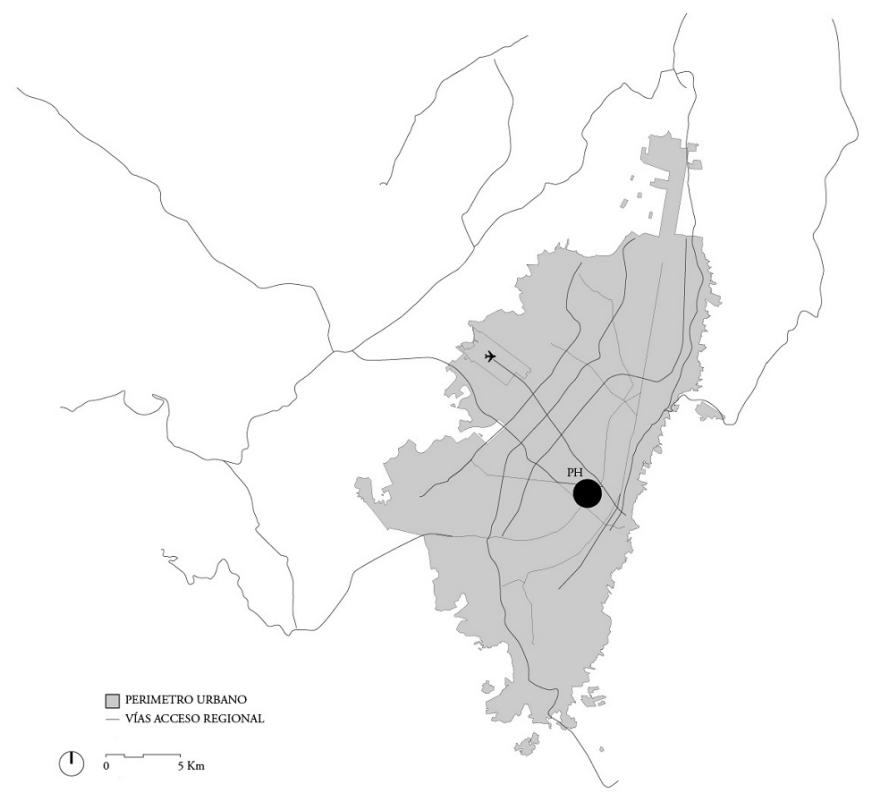

FUENTE: ELABORACIÓN PROPIA

Lo anterior implicó que se destinaran a ello importantes esfuerzos de distintas entidades municipales. Metrovivienda, entidad encargada hasta ese momento de promover la construcción y adquisición de vivienda de interés prioritario, tuvo un papel protagónico en el desarrollo del proyecto, a diferencia de su participación en otros de los proyectos del PVG que tuvieron lugar en Bogotá.

En el caso de Plaza de la Hoja, por una decisión más política, ya que este se convirtió en el programa bandera de esa administración (Bogotá Humana), Metrovivienda intervino de manera más aguda en el proyecto (...). En Plaza de la Hoja tuvo un papel más protagónico y el proceso fue un poco atípico. En este sentido, realizó acompańamiento a todo el tema de adjudicación de la vivienda (cuando ya se 
tenían a las familias seleccionadas), de entrega de las viviendas y también en la estrategia para la vinculación y restablecimiento del tejido social de las personas en su nuevo territorio. También hubo un trabajo de acompańamiento social en el barrio Cundinamarca, en la medida que ellos tenían un rechazo agudo sobre el proyecto.

(Entrevista funcionario Metrovivienda, septiembre 2016)

Adicional a la gestión realizada en el ámbito social - tanto previa como posterior al desarrollo del proyecto-, Metrovivienda hizo las veces de propietario del predio (una vez que el IDU lo transfirió) y promotor. De esta manera, dicha entidad llevó a cabo actividades como solicitar la licencia de urbanización; realizar de manera simultánea un concurso para seleccionar el diseño arquitectónico del proyecto (en conjunto con la Secretaría Distrital de Ambiente y apoyado por la Sociedad Colombiana de Arquitectos); la constitución de un esquema fiduciario con el objeto de verificar tanto el proceso de complementación de los estudios y diseńos como la posterior construcción de las obras de urbanismo y de las viviendas (Contraloría de Bogotá D.c., 2014).

El proyecto ganador del concurso realizado, y que se llevó a la construcción, es reconocido por varios aspectos, entre los cuales se cuenta la flexibilidad del diseño de la vivienda, que permite la adaptación de diversos espacios de acuerdo con los requerimientos de cada grupo familiar, pudiendo destinarse algunos para actividades productivas (Alcaldía Mayor de Bogotá, Metrovivienda, 2014). Se resalta también, en la disposición de la agrupación de vivienda, que el proyecto dispone de circulaciones tanto horizontales como verticales, que conforman patios y galerías para el encuentro y relacionamiento comunal (Figura 2). Por último, respecto de la relación del proyecto con su entorno, se menciona como un aspecto valioso la permeabilidad del primer piso, de carácter público. En este se plantean usos dotacionales y complementarios al residencial (especialmente comercio), que buscan conectar el espacio central del proyecto con la ya existente Plaza de la Hoja (Alcaldía Mayor de Bogotá, Metrovivienda, 2014).

\section{FIgURA 2 | Circulaciones comunales segundo piso Plaza de la Hoja, Bogotá}

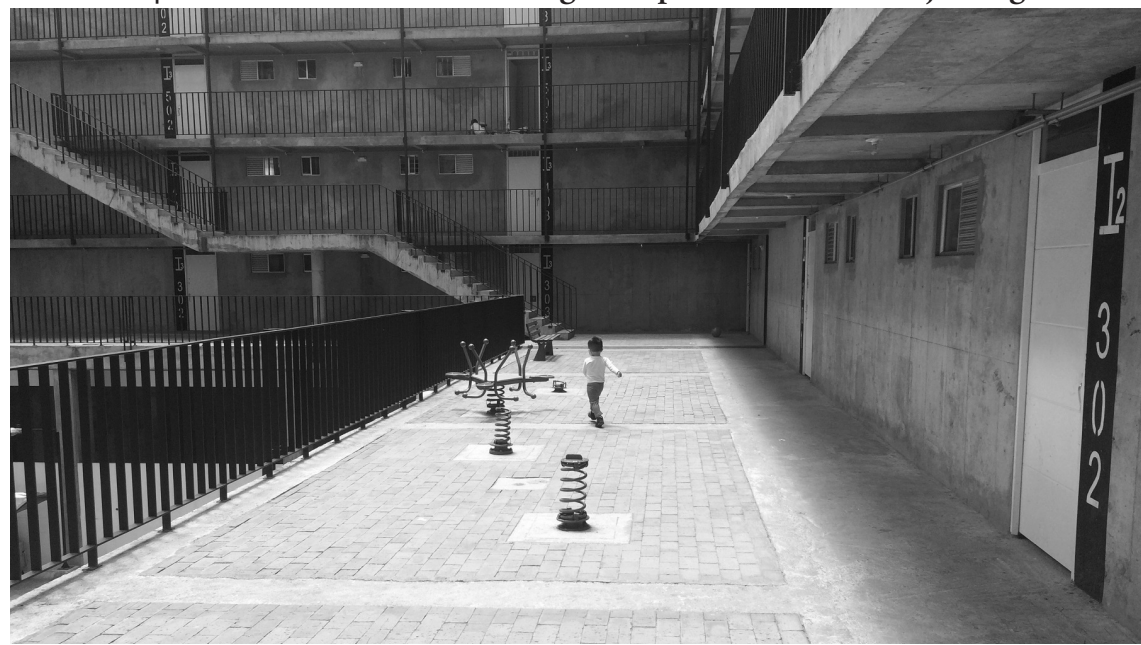

FUENTE: FOTOGRAFÍA AUTORES 
Sin embargo, en el análisis realizado al proyecto se encontraron algunos aspectos que lo desvían de su concepción y propósito original. Por un lado, fueron efectuadas modificaciones a su diseño original, especialmente en lo que respecta a la configuración del primer piso del proyecto, que desdibujan su carácter público y de encuentro colectivo. ${ }^{2}$ Entre tales modificaciones, se encontró que en el espacio central del conjunto residencial se ubicaron los espacios comunales de parqueo vehicular, cuando se pretendía que fuera de disfrute peatonal (Figura 3). En tres de las torres se desarrollaron unidades de viviendas en los primeros pisos, se ubicaron algunos espacios de uso comunal, y se modificaron y redujeron los espacios correspondientes al uso comercial (con base en el análisis del proyecto inicial en Alcaldía Mayor de Bogotá, Metrovivienda, 2014; y de la planta de primer piso aprobada para su construcción en Licencia Res 14-2-09 de la Curaduría Urbana N² 2, 2014).

FIgURA 3 | Espacio público y comunal Plaza de la Hoja, Bogotá
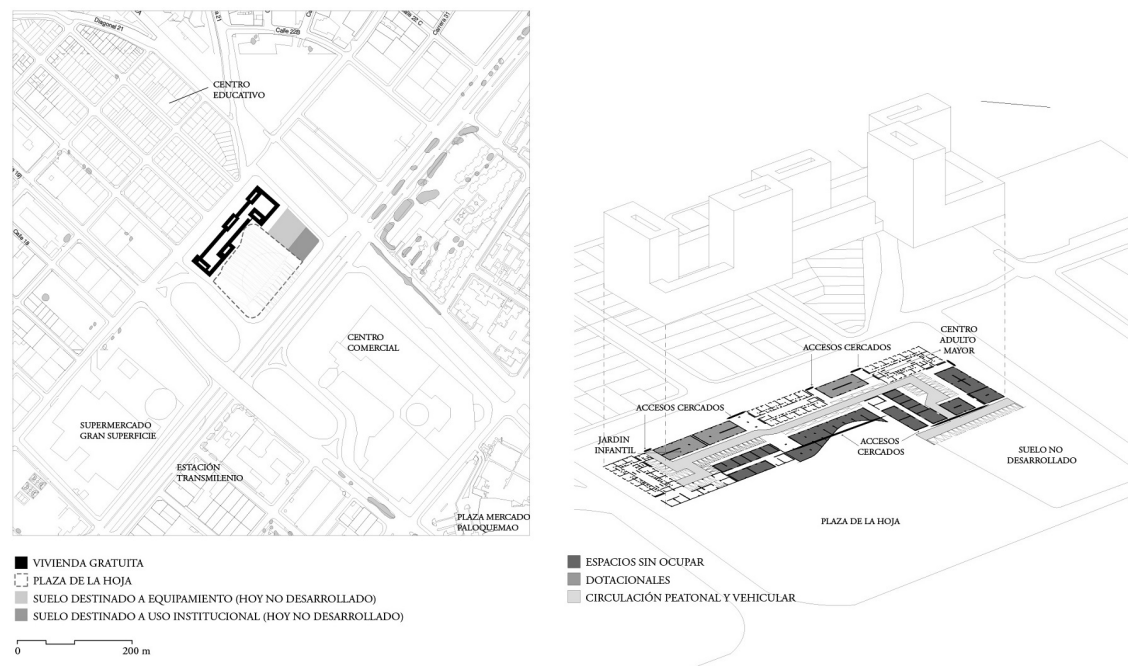

FUENTE: ELABORACIÓN PROPIA

Al momento de la visita efectuada al proyecto a poco más de un año de entregadas las viviendas, estos espacios comerciales no se encontraban adecuados ni funcionando, ni había claridad en los mecanismos para su adjudicación y gestión. De igual manera, en algunos de los espacios destinados al uso comercial se ubicaron equipamientos públicos: un jardín infantil y un centro día para adultos de la tercera edad (puestos en funcionamiento en 2016); además la comunidad había cercado el espacio central del proyecto para su disfrute comunal.

Se relaciona lo anterior con la insatisfacción frente al proyecto por parte de algunos de sus residentes, que mencionan la existencia de conflictos vecinales

2 Se diferencian los espacios colectivos como espacios de disfrute público y de la ciudadanía, de aquellos comunales, que son para el uso de los habitantes de un conjunto residencial/barrio determinado, dependiendo de cada caso. El carácter de estos últimos es privado. 
derivados del funcionamiento de los espacios del primer piso, y la desinformación de la comunidad en lo que se refiere a su gestión (Figura 4). En cuanto a satisfacción, la mayoría de los residentes encuestados, el $91 \%$, se encuentra a gusto con su vivienda de manera contundente. Sin embargo, este porcentaje disminuye considerablemente, al $73 \%$, en lo que respecta a su nivel de satisfacción con el conjunto de vivienda Plaza de la Hoja (encuesta residentes Plaza de la Hoja, agosto 2016). Especialmente se hace referencia a la inacabada culminación del proyecto en lo que respecta a la instalación de la totalidad de los ascensores, a locales que no han sido asignados, parqueaderos faltantes y realizados en las zonas de tránsito peatonal del proyecto, y a la falta de entrega oficial de los espacios que consideran comunales por parte de Metrovivienda. (Taller focal líderes Plaza de la Hoja, agosto 2016)

Figura 4 | Primer piso Plaza de la Hoja, Bogotá. Espacios de parqueo. Comercios no adjudicados

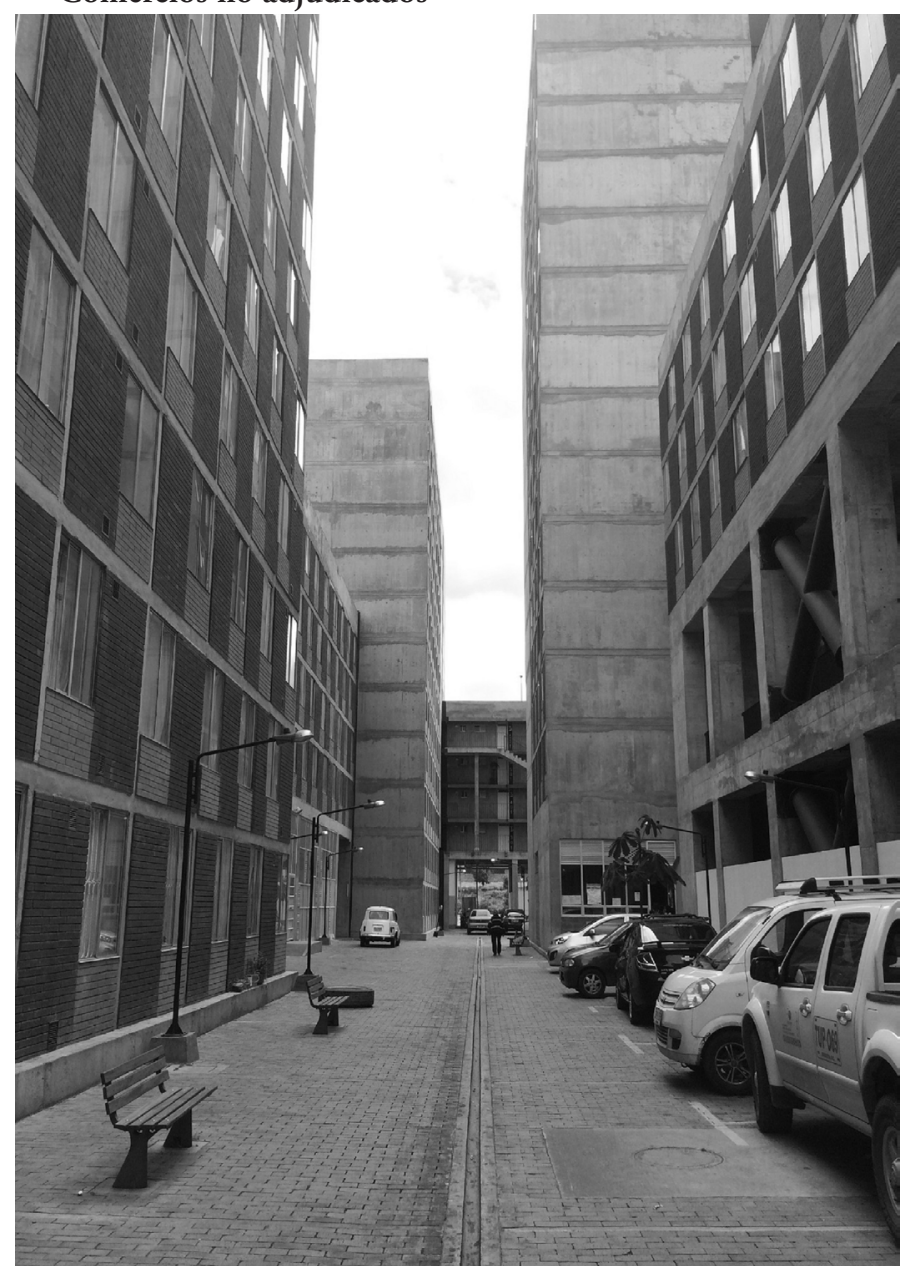

FUENTE: FOTOGRAFÍA AUTORES 
No obstante, es importante resaltar que la comunidad reconoce el esfuerzo institucional, tanto municipal como nacional, en lo que respecta a la entrega de viviendas gratuitas en una zona estratégica de la ciudad:

(E)l gobierno de Santos fue el que instauró este programa de viviendas gratuitas y aquí en Bogotá se desarrollaron varios proyectos. Al alcalde Petro se le puede abonar que fue uno de los abanderados para que la gente, sobre todo víctimas de la violencia, fuéramos beneficiarios de ese programa, y que los sitios donde nos ubicaran fueran sitios no periféricos, sino más bien en el interior de la ciudad (...)

y pues lo valoramos. (Taller focal líderes Plaza de la Hoja, agosto 2016)

Sin lugar a duda, el análisis refleja que el proyecto acercó los hogares beneficiados a fuentes de servicios y de transporte. Lo anterior aparece evidenciado en que al menos tres cuartos, $84,85 \%$ de las personas del grupo encuestado, reconoce de manera significativa esta condición de proximidad física, y menos de la mitad, un $35 \%$, reporta haber tenido dificultades para adaptarse a este nuevo entorno (encuesta residentes Plaza de la Hoja, agosto 2016).

Sin embargo, tal proximidad no ha implicado en todos los casos el acceso a estos servicios, como por ejemplo en lo que respecta a colegios, por falta de cupos en los establecimientos de la zona, o el surgimiento de situaciones de exclusión social en estos espacios (taller focal líderes Plaza de la Hoja, agosto 2016). También, más de la mitad de los encuestados, $75,76 \%$, considera que el proyecto no ha implicado proximidad a parques y zonas verdes. Finalmente, e igual de relevante, el análisis revela que un poco más de la mitad de los encuestados, el 65\%, no reconoce que las oportunidades laborales para los miembros de su familia se hayan incrementado con respecto a su contexto anterior (encuesta residentes Plaza de la Hoja, agosto 2016). Al respecto, también se menciona la falta de entidades que acompañen a la comunidad, y especialmente la ausencia de apoyo para la formación de capacidades que habiliten el acceso al mercado laboral (taller focal líderes Plaza de la Hoja, agosto 2016).

\section{Villas de San Pablo (Barranquilla)}

El proyecto Villas de San Pablo (Figura 5), a diferencia de Plaza de la Hoja, se ejecuta mediante la modalidad de desarrollo privada contemplada por el PVG en el periodo 2013-2014. El proyecto se encuentra en Barranquilla a las afueras de la ciudad, en medio de un suelo de expansión urbana, a aproximadamente 12 kilómetros de distancia del centro. El proyecto se construyó en 52 torres de cinco pisos, que se distribuyen en dos manzanas, entre las cuales se destina espacios a parqueo, recorrido peatonal y otros de uso comunal. En el marco del programa se desarrollaron 832 unidades de vivienda, 630 en una manzana y 192 en otra de menor tamaño. 
FIgura 5 | Localización de Villas de San Pablo, Barranquilla

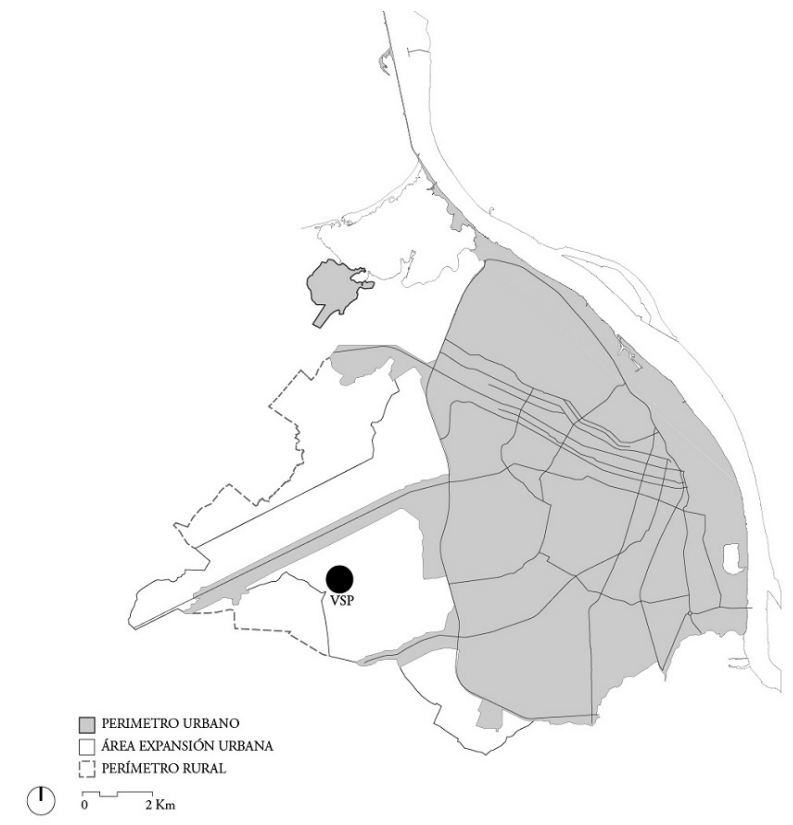

FUENTE: ELABORACIÓN PROPIA

Las dos manzanas donde el proyecto fue desarrollado pertenecían a un ámbito de planificación y gestión más extenso: un macroproyecto de interés social. ${ }^{3} \mathrm{Su}$ localización en un polígono de desarrollo mayor es, en primera instancia, una de las particularidades de este proyecto. Permitió que las manzanas de vivienda gratuita se contextualizaran como parte de una unidad funcional más amplia, y que las unidades de vivienda se desarrollaran en medio de una estructura urbana de alcance local/zonal en lo que respecta a la oferta de vías y de suelos para el desarrollo de espacios públicos, de recreación e infraestructura social. Este es uno de los aspectos que valoran los agentes participantes en la gestión del proyecto, que concibieron el desarrollo de la vivienda gratuita en medio de un barrio en proceso de consolidación (entrevista Fundación Mario Santo Domingo, julio 2016).

No obstante, para el momento del análisis del proyecto — dos años después de construido- esta red de espacios públicos e infraestructura social no estaba completamente consolidada. Junto con las viviendas, se habían construido un Punto Vive Digital (2013), un Centro de Desarrollo Integral (2014), y de manera posterior a su construcción, un Comando de Acción de Atención Inmediata (2015), una biblioteca ( $s i n$ datos de fecha de su desarrollo) y estaban en proceso de desarrollo un megacolegio y un Punto de Atención en Salud Oportuna (ambos inaugurados en 2018).

Operación de desarrollo urbano y de vivienda de interés social y prioritaria promovida por el nivel central de gobierno, a cargo del Ministerio de Vivienda, Ciudad y Territorio, cuyo objetivo yace en la ampliación de la oferta de suelo urbanizado para el desarrollo de estos tipos de vivienda. 
FIGURA 6 | Espacio público y comunal Villas de San Pablo, Barranquilla

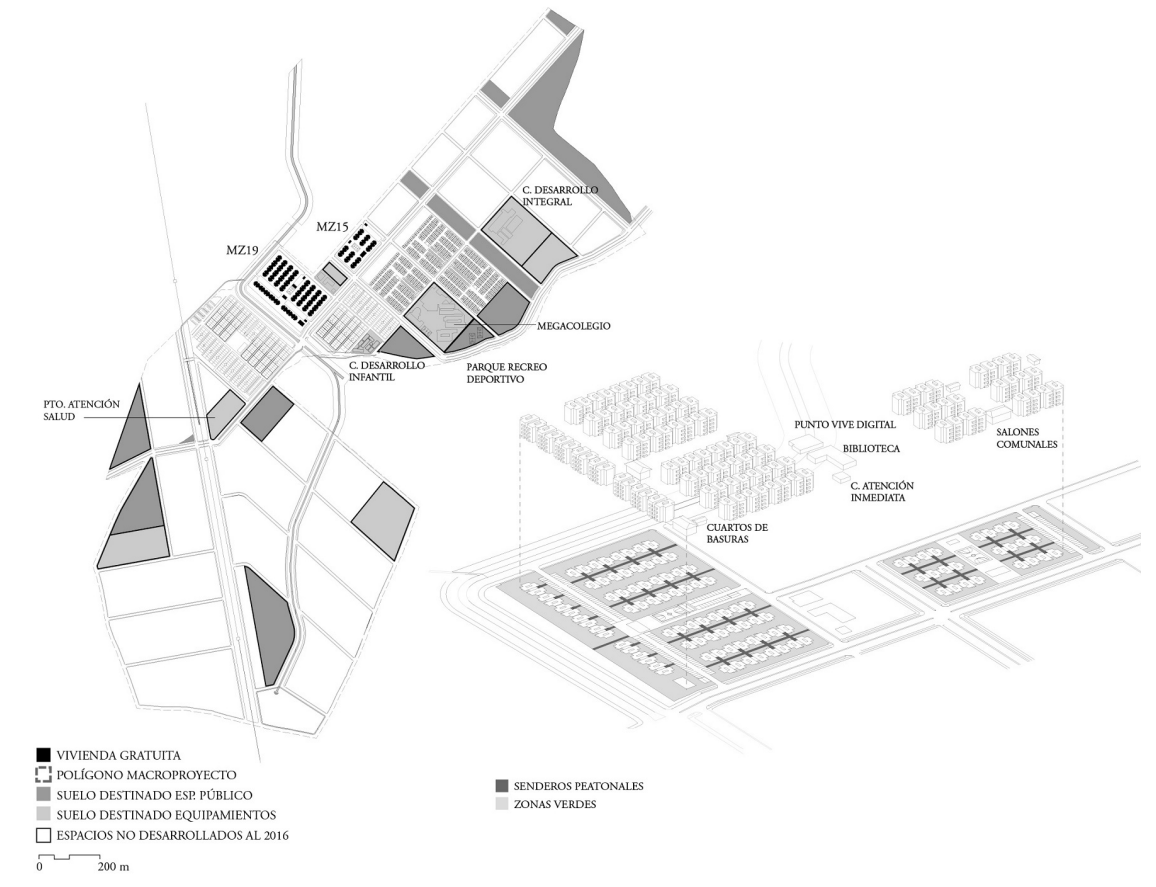

FUENTE: ELABORACIÓN PROPIA

Si bien las dos manzanas de vivienda gratuita se beneficiaron de esta estructura urbana en proceso de desarrollo, el macroproyecto de interés social nacional y otras unidades de nuevas viviendas sociales que allí se han desarrollado, se han visto beneficiados por el PVG. El mismo gobierno nacional, en el marco del PVG priorizó siete macroproyectos —entre los que se encontraba Villas de San Pablo- para el desarrollo de equipamientos de educación, bienestar social, cultura y salud, entre otros (Ministerio de Vivienda, Ciudad y Territorio, 2014b). Desde la gestión del macroproyecto se asocia la llegada de la población beneficiada por el PVG, a la construcción de la oferta de infraestructura social que antes no se había desarrollado en Villas de San Pablo (Figura 6):

Cuando llega Vivienda Gratis, Villas de San Pablo tenía alrededor de 400 personas, y llegan 832 familias; ese día se dispara la población (...) la dinámica del territorio es totalmente distinta. Llega mayor cantidad de población, había 600 niños, luego 1400 , lo cual ya genera otros intereses. Se venía luchando muchos ańos por un colegio, pero se necesitaba una cobertura de 1440 cupos; entonces se construye el colegio y puesto de salud. Al completar ese cupo de personas necesarias, se gestionan aún más los servicios. (Entrevista Fundación Mario Santo Domingo, julio 2016)

Otra de las particularidades con que contó este proyecto fue la vinculación de un agente de carácter privado que ha estado presente a lo largo de diversas etapas de su 
desarrollo y que ha permanecido en el territorio tras la adjudicación de las viviendas: la Fundación Mario Santo Domingo. Esta era inicialmente propietaria del suelo donde se desarrolló el macroproyecto, y actuó como gestora de este. En el marco del PVG, se asoció con la constructora Prodesa a través de una unión temporal para el diseño y la construcción de las unidades de vivienda, ya que esta última empresa contaba con la experiencia en construcción requerida para presentar propuestas al programa (entrevista Fundación Mario Santo Domingo, julio 2016; Fideicomiso PVG, 2012).

Una vez aprobado y construido el proyecto de vivienda, la Fundación inició diversas actividades con las familias beneficiadas por el programa, en el marco de lo que la organización denomina la ruta vaAs: Vinculación, Adaptación, Acompañamiento y Salida. En la etapa de vinculación se desarrollaron diversos talleres con los hogares, previamente a que se instalaran en la vivienda, donde se les informó de las características del proyecto y sus condiciones de acceso a servicios complementarios, así como aspectos relevantes para la convivencia comunal, entre otros temas. En el proceso de adaptación de las familias, la Fundación apoyó a la comunidad tanto en el establecimiento de una estructura de comités que permitiera su organización y convivencia, como en el planteamiento de un plan para el desarrollo comunitario (entrevista a la Fundación Mario Santo Domingo, julio 2016).

Las labores de acompañamiento y gestión social señaladas, y su incidencia en la adaptación de las familias beneficiadas, son reconocidas de manera positiva por otros agentes que han estado involucrados en el proceso. Dichos agentes valoran especialmente la positiva influencia de la Fundación en la socialización y apropiación del manual de convivencia por parte de los nuevos residentes, labor que no se ha llevado a cabo en otros proyectos de Barranquilla por parte de los constructores. Se asumen estas actividades como experiencias únicas de participación en el marco del PVG (entrevista funcionarios Secretaría de Planeación Barranquilla, julio 2016). Líderes de la comunidad reconocen que la labor de la Fundación ha sido efectiva en la gestión de ayudas y otros programas sociales, gracias a la capacidad que la entidad tiene para canalizar iniciativas de diversas agrupaciones, de acuerdo con las necesidades de la comunidad de Villas de San Pablo (taller focal líderes Villas de San Pablo, julio 2016).

Otros aspectos de relevancia surgieron en lo que se refiere a la satisfacción de los usuarios con el proyecto y su proceso de desarrollo. Aunque en Villas de San Pablo el nivel de satisfacción con la vivienda, revelado por las encuestas realizadas a la población residente es similar al encontrado en Plaza de la Hoja (94\% de los encuestados satisfechos, frente al 91\% en Plaza de la Hoja), es de notar que en lo que se refiere a la satisfacción de los residentes frente a su conjunto residencial y espacios comunales, no dista demasiado del obtenido con la vivienda ( $90 \%$ de los encuestados manifiesta estar satisfechos con el proyecto de vivienda, cifra que en caso de Plaza de la Hoja solo llegaba al 73\%). Lo anterior puede atribuirse a la configuración clara de espacios comunales y públicos con la que cuenta el proyecto (Figura 7), y a las ya mencionadas labores de socialización del manual de convivencia, que clarifica las responsabilidades de los residentes frente a estos espacios (encuesta residentes Villas de San Pablo, julio 2016). 
FIGURA 7 | Espacios comunales Villas de San Pablo, Barranquilla

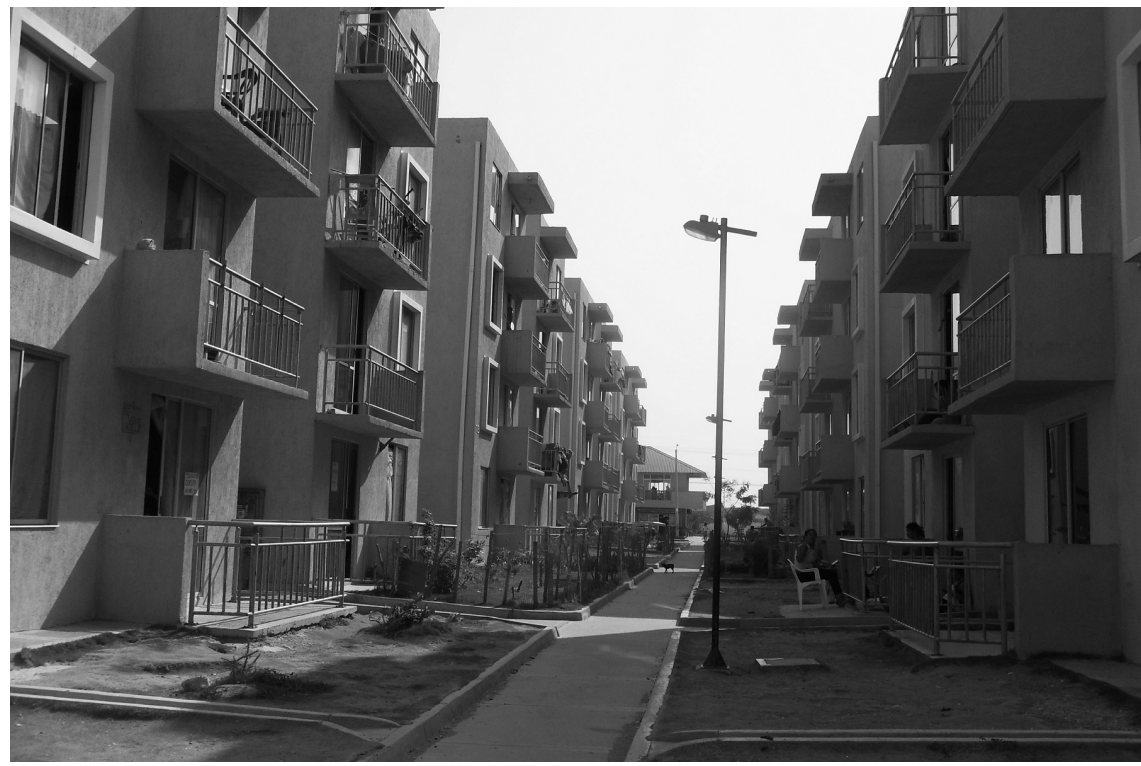

FUENTE: FOTOGRAFÍA AUTORES

No obstante, una relativamente alta proporción de la población beneficiada ha encontrado dificultades para adaptarse al nuevo entorno, las que pueden encontrar asidero en las condiciones de localización del proyecto, que se encuentra distante de la ciudad y su centro. Lo anterior tiene efecto en la disminución del acceso al mercado laboral y redes de información en este aspecto, por parte de la población beneficiada:

Aunque tengo mi vivienda, que es una bendición, no tenía dónde montar mi peluquería. Sin embargo, me vine y la monté, pero no dio, porque en un apartamento, el gasto de servicio, no hay los espacios adecuados y hubo muchos inconvenientes. Aquí a muchas familias les ha costado lo que es la fuente de empleabilidad, porque todo queda lejos, hay que pagar cuatro transportes para llegar, vas a ganar un mínimo y vas a pagar como la mitad en transporte. (Taller focal líderes Villas de San Pablo, julio 2016)

Esto también encuentra soporte en algunos de los datos encontrados en la encuesta realizada a los residentes de vivienda gratuita de Villas de San Pablo, teniendo en cuenta que el $43 \%$ de la población encuestada encontró dificultades para adaptarse al nuevo entorno. Tales dificultades también pueden estar referidas a los equipamientos e infraestructura social, que no se encontraban funcionando al momento del análisis (aproximadamente la mitad de los encuestados considera no tener acceso a servicios básicos, entre los que se menciona de manera reiterada el acceso a salud). En relación con dificultades de acceso al mercado laboral, el 74\% de los encuestados considera que no se han incrementado las oportunidades laborales para los miembros de su 
familia desde su llegada a Villas de San Pablo, con respecto a su contexto de residencia anterior (Encuesta residentes Villas de San Pablo, julio 2016).

No obstante, la distancia espacial del proyecto con respecto a otros sectores de la ciudad ha mostrado que, en algunos casos, puede conducir también al debilitamiento de redes de solidaridad familiar:

En lo referente a mis padres, ahora no es lo mismo, no tengo plata para ir a verlos; me demoro hasta meses $\sin$ ir a verlos, porque se paga mucho transporte para ir a verlos allá. Si tengo los 10.000 o 12.000 pesos para un taxi, no tengo para la energía o para mis hijas. Las cosas cambian mucho, porque ya no hay plata para ir a verlos. A mí me gustaría ir a verlos todos los fines de semana, pero no se puede. Las cosas han cambiado muchísimo. (Taller focal líderes Villas de San Pablo, julio 2016)

Por último, en cuanto a la percepción de cercanía frente a otros espacios de importancia urbana, el $34 \%$ de los encuestados considera que el proyecto no está próximo a espacios verdes, parques y otros espacios de recreación (encuesta residentes Villas de San Pablo, julio 2016). Aunque para el momento del análisis el parque deportivo recreativo todavía estaba en proceso de desarrollo, los espacios de reunión comunal (que son, en estricto sentido, de carácter privado) son reconocidos por los residentes como espacios de disfrute colectivo.

En Tabla 2, los resultados de la encuesta para los dos proyectos analizados.

TABLA 2 Preguntas de percepción y satisfacción de residentes proyectos analizados

\begin{tabular}{|c|c|c|c|c|c|c|c|c|c|c|c|c|}
\hline \multirow[t]{3}{*}{$\begin{array}{l}\text { Preguntas } \\
\text { realizadas } \\
\text { encuesta a } \\
\text { residentes } \\
\text { / Proyec- } \\
\text { tos }\end{array}$} & \multicolumn{2}{|c|}{$\begin{array}{l}\text { ¿A usted y } \\
\text { su familia } \\
\text { les gusta su } \\
\text { vivienda? }\end{array}$} & \multicolumn{2}{|c|}{$\begin{array}{l}\text { ¿Se encuentran } \\
\text { satisfechos con } \\
\text { este proyecto } \\
\text { de vivienda? }\end{array}$} & \multicolumn{2}{|c|}{$\begin{array}{l}\text { ¿Usted o su } \\
\text { familia encon- } \\
\text { traron alguna } \\
\text { dificultad para } \\
\text { adaptarse a } \\
\text { este nuevo } \\
\text { entorno? }\end{array}$} & \multicolumn{2}{|c|}{$\begin{array}{l}\text { ¿Vivir en este } \\
\text { conjunto } \\
\text { residencial les } \\
\text { ha generado } \\
\text { proximidad } \\
\text { a servicios } \\
\text { básicos? }\end{array}$} & \multicolumn{2}{|c|}{$\begin{array}{l}\text { ¿Vivir en este } \\
\text { conjunto } \\
\text { residencial les } \\
\text { ha generado } \\
\text { proximidad } \\
\text { a espacios y } \\
\text { corredores ver- } \\
\text { des, parques y } \\
\text { otros espacios } \\
\text { de recreación? }\end{array}$} & \multicolumn{2}{|c|}{$\begin{array}{l}\text { ¿Considera } \\
\text { que se incre- } \\
\text { mentaron las } \\
\text { oportunidades } \\
\text { laborales para } \\
\text { los miembros } \\
\text { de su familia } \\
\text { (puestos de } \\
\text { trabajo) frente } \\
\text { a su contexto } \\
\text { anterior? }\end{array}$} \\
\hline & \multicolumn{12}{|c|}{ Porcentajes } \\
\hline & Sí & No & Sí & No & Sí & No & Sí & No & Sí & No & Sí & No \\
\hline $\begin{array}{l}\text { Plaza de } \\
\text { la Hoja }\end{array}$ & 91 & 9 & 73 & 27 & 35 & 64 & 85 & 15 & 24 & 76 & 33 & 62 \\
\hline $\begin{array}{l}\text { Villas de } \\
\text { San Pablo }\end{array}$ & 94 & 6 & 89 & 9 & 43 & 57 & 47 & 49 & 62 & 34 & 26 & 74 \\
\hline
\end{tabular}

NOtA: ALGUNAS SAlVEdAdes: LA ENCUESTA SE REALizó A RESIDENTES DE LOS PROYECTOS ANALIZADOS, PERO LAS PREGUNTAS ESTÁN PLANTEADAS CON BASE EN LOS HOGARES COMO UNIDAD DE ANÁLISIS. ASIMISMO, SE INCLUYEN AQUELLAS PREGUNTAS QUE SE CONSIDERAN RELEVANTES PARA DAR CUENTA DE ASPECTOS DE SATISFACCIÓN Y PERCEPCIÓN QUE REMITEN A LA GESTIÓN DE LOS PROYECTOS. FINALMENTE, LAS RESPUESTAS DADAS POR LOS RESIDENTES PODÍAN SER AMPLIADAS DE MANERA NARRATIVA; DICHOS RESULTADOS SE HAN CONTRASTADO CON AQUELLOS OBTENIDOS DE LAS ENTREVISTAS A LÍDERES Y LOS TALLERES FOCALES.

FUENTE: ELABORACIÓN PROPIA 


\section{Conclusiones}

En lo que respecta al Programa de Vivienda Gratuita (PVG), se concluye que el gobierno nacional dispuso de manera expedita recursos organizacionales y financieros en diferentes niveles administrativos, para lograr el desarrollo de 100.000 viviendas en alrededor de cuatro años. Sin embargo, se considera que la rapidez de este proceso implicó sacrificios en las condiciones de habitabilidad de la población beneficiada, ello derivado de lo encontrado en los dos casos estudiados. Aunque no se pretende con el análisis generalizar sobre los efectos del programa, sí se puede dar luces sobre su proceso de implementación en ciudades principales del país, que se considera son aquellas con esquemas y recursos de gestión más robustos y donde finalmente se desarrolló alrededor de un tercio de su oferta.

En relación con su diseño y el de sus arreglos normativos, se encontró la disposición de un marco de reglamentación amplio. No obstante, se detectaron dos aspectos principales en la ejecución de los proyectos estudiados que parecen escapar al diseño inicial del programa, relacionados con los procesos de coordinación desarrollados en el marco de este, y sus efectos en el estado de desarrollo de los proyectos.

En primera instancia, está la ausencia de lineamientos para el desarrollo de equipamientos e infraestructura social asociados a los proyectos de vivienda en el momento de su creación. Tales lineamientos se definieron cuatro ańos después de entrado en vigencia el programa, cuando ya había proyectos de vivienda desarrollados y se evidenciaban problemas de convivencia y habitabilidad, especialmente por la falta de infraestructura social (es el caso de Villas de San Pablo). En lo que se refiere al desarrollo de la infraestructura social, se encuentran contrastes en el grado de participación, involucramiento y capacidad de gestión de los diferentes niveles de gobierno y entidades participantes. Lo anterior deriva en condiciones muy diferenciadas de ofertas sociales y beneficios brindados a los hogares residentes de los proyectos.

En segunda instancia, aunque el programa definió de manera detallada las competencias otorgadas a cada uno de los agentes involucrados en su ejecución, se encuentran algunas indefiniciones en la asignación de responsabilidades, especialmente en lo que respecta al desarrollo de actividades de seguimiento y acompańamiento ex post de la población beneficiada. Lo anterior deriva en la actuación de algunas entidades por fuera de su misión o en la ausencia de realización de dichas actividades en otros proyectos. Este es el caso de Metrovivienda en Bogotá, entidad a la cual, dado su carácter de banco de suelos, no corresponde la misión que le ha sido asignada en el acompañamiento ex post de las familias beneficiadas por el programa, considerando una capacidad de gestión limitada para el desarrollo de estas actividades.

En lo que respecta a la articulación del programa con los instrumentos dispuestos en el marco jurídico-urbanístico colombiano y de planificación territorial, coinciden los dos casos estudiados en que no han hecho uso y aplicación de instrumentos de gestión y financiación urbana para la consecución del suelo de los proyectos de vivienda. En Plaza de la Hoja el suelo lo aportó una entidad pública, y en Villas de San Pablo, un privado a quien el programa retribuyó su valor en concordancia con 
la modalidad de gestión privada. En este último caso, sin embargo, el proyecto sí se desarrolló en el marco de un ámbito de planificación que le brindó estructura urbana y de cobertura barrial/zonal, aunque en una localización periférica.

Esta falta de articulación entre los proyectos y el marco jurídico-urbanístico colombiano podría estar contribuyendo a la postulación de suelos periféricos por parte de actores tanto privados como públicos (teniendo como excepción lo sucedido en Bogotá), que deriva también en bajas coberturas tanto de servicios complementarios y dotacionales como de transporte público. Es de notar que esta oferta periférica adicionalmente podría ir en contravía de las disposiciones de planificación de los gobiernos locales e implicar para ellos costos adicionales, destinados a servir los predios donde los proyectos se desarrollan.

En cuanto a las acciones llevadas a cabo para la gestión social en el marco del programa, se encuentra que los mecanismos planteados para la participación y empoderamiento de la población son incipientes. Por un lado, la participación es entendida por los agentes institucionales como la socialización de aspectos de relevancia para la comunidad y su convivencia. Por otro, la población beneficiada de los dos proyectos reporta una baja incidencia de los programas sociales de acompañamiento y acciones de seguimiento implementadas en el marco del programa. Especialmente se menciona la ausencia de programas de apoyo y formación de competencias para la inserción laboral, y la falta de articulación con redes empresariales y requerimientos del mercado laboral de los existentes.

En conclusión, se considera que la gestión de programas de vivienda social debe abordarse de manera integral, con miras a brindar condiciones que faciliten la integración social de los hogares beneficiados al ámbito urbano. Esto implica disponer lineamientos tanto para la coordinación interinstitucional, como para la definición clara de competencias de los actores involucrados en su desarrollo, la gestión social y seguimiento de los hogares beneficiados y la gestión urbana de los proyectos que cobijan. Especialmente, los procesos de gestión urbana deben contribuir a la integración social a través del desarrollo de servicios complementarios a la vivienda, de infraestructura social de carácter local y urbano, y de una clara disposición de los espacios comunales que facilite los procesos de convivencia de los residentes. Estos aspectos también deben darse con miras a fortalecer la apropiación de la vivienda social por parte de sus residentes en el tiempo.

\section{Referencias bibliográficas}

Alcaldía Mayor de Bogotá, Metrovivienda. (2014). Plaza de la Hoja: Revitalización con vivienda digna en Bogotá Humana. Metrovivienda.

Alcaldía Mayor de Bogotá, Secretaría de Planeación \& Secretaría de Hábitat. (2014). El plan urbano del centro ampliado de Bogotá. Una estrategia para la revitalización urbana. Alcaldía Mayor de Bogotá / Bogotá Humana. https://docplayer.es/21146724-El-planurbano-del-centro-ampliado-de-bogota.html 
Arango, G. (2001). La vivienda en Colombia en el cambio de siglo. Herencias y retos. En P. C. Brand (Ed.), Trayectorias urbanas en la modernización del Estado en Colombia (pp. 227263). тм Editores / Universidad Nacional de Colombia Sede Medellín. http://bdigital. unal.edu.co/9830/1/gaeVivCol.pdf

Bedoya Ruiz, A., Agudelo Rodríguez, C. F., Ramos Calonge, H. \& Juliao Vargas, C. S. (2018). Vivienda social en Colombia. Metodología para su evaluación integral. Ediciones Unisalle.

Brain, I., Cubillos, G. \& Sabatini, F. (2007). Integración social urbana en la nueva política habitacional de Chile. Pontificia Universidad Católica de Chile.

Camacho-Cardona, M. (1998). Diccionario de arquitectura y urbanismo. Trillas.

Cohen, E. \& Franco, R. (2005). Gestión social. Cómo lograr eficiencia e impacto en las politicas sociales. Siglo XXI.

Comisión Económica para América Latina y el Caribe (CEPAL). (2003). Gestión urbana para el desarrollo sostenible en América Latina y el Caribe [LC/G.2203-P]. R. Jordán \& D. Simioni (Comps.). CEPAL / Naciones Unidas. https://repositorio.cepal.org/bitstream/ handle/11362/2376/1/S2003000_es.pdf

Contraloría de Bogotá D.c. (2014). Metrovivienda. Informe de auditoría. Modalidad regular. Periodo auditado 2013. Plan de Auditoría Distrital (PAD) 2014. Dirección Sector Hábitat y Ambiente [Informe preliminar]. https://bit.ly/34SdfeE

Cuervo, N. \& Jaramillo, S. (2010). Dos décadas de política de vivienda en Bogotá apostando por el mercado. En S. Jaramillo (Ed.), Bogotá en el cambio de siglo: promesas y realidades (pp. 249-292). Olacchi.

Curaduría Urbana $\mathrm{N}^{\circ}$ 2. (2014). Plano de referencia licencia de construcción (Res 14-2-09). Consultado en el Archivo Central de la Secretaría de Planeación Distrital.

Fernández-Ballesteros, R. (Ed.). (2011). Evaluación de programas. Una guia práctica en ámbitos sociales, educativos y de salud. Síntesis.

Fideicomiso Programa de Vivienda Gratuita. (2012). Convocatoria PVG. Acta 027. https://bit. $1 y / 3276 \mathrm{t} 2 \mathrm{Y}$

Kesteloot, C., Murie, A. \& Musterd, S. (2006). European cities: neighborhood matters. En S. Musterd, A. Murie \& C. Kesteloot (Eds.), Neighbourhoods of poverty: urban social exclusion and integration in comparison (pp. 219-238). Palgrave Macmillan.

Kleinman, M. P. (1996). Housing, welfare and the State: A comparative analysis of Britain, France, and Germany. Edward Elgar.

Leal-Maldonado, J. (2005). Desigualdad residencial y sistema de Bienestar en España. En J. Ruiz-Huerta Carbonell (Coord.), Políticas públicas y distribución de la renta (pp. 391436). Fundación BBva.

Maclennan, D. (2005). Housing policies: new times, new foundations. Reporte de investigación comisionada por la Joseph Rowntree Foundation. http://cprn3.library.carleton.ca/ doc.cfm?doc $=1189 \& \mathrm{l}=\mathrm{en}$

Marcuse, P. (2005). Enclaves yes, ghettos no. Segregation and the State. En D. Varady (Ed.), Desegregating the city. ghettos, enclaves \& inequality (pp. 15-31). University of New York Press.

Ministerio de Vivienda, Ciudad y Territorio (муст), Gobierno de Colombia. (2014a). Colombia: Cien años de políticas habitacionales. мvст. 
Ministerio de Vivienda, Ciudad y Territorio, Gobierno de Colombia. (2014b, marzo 14). Minvivienda inicia proceso de acompañamiento social a 2 proyectos de vivienda gratuita en Medellin. Noticias. https://bit.ly/2I1ItaF

Ministerio de Vivienda, Ciudad y Territorio, Gobierno de Colombia. (2015a). Especial de 100 mil Viviendas $100 \%$ subsidiadas. https://bit.ly/3848wbT

Ministerio de Vivienda, Ciudad y Territorio, Gobierno de Colombia. (2015b, febrero 25). Resolución 119 de 2015. http://www.alcaldiabogota.gov.co/sisjur/normas/Norma1. jsp?i=61119

Morales-Schechinger, C. (2005). Políticas de suelo urbano, accesibilidad de los pobres y recuperación de plusvalías. En M. Carmona, Grandes proyectos urbanos. La respuesta de 25 ciudades (pp. 94-112). Infinito.

Musterd, S. (2003). Segregation and integration: a contested relationship. Journal of Ethnic and Migration Studies, 29(4), 623-641. https://doi.org/10.1080/1369183032000123422

Musterd, S. (2005). Social and ethnic segregation in Europe: levels, causes, and effects. Journal of Urban Affairs, 27(3), 331-348. https://doi.org/10.1111/j.0735-2166.2005.00239.x

Organización de las Naciones Unidas (ONU)-Hábitat \& Banco de Desarrollo de América Latina (CAF). (2014). Construcción de ciudades más equitativas. Políticas públicas para la inclusión en América Latina. onU-Hábitat. https://bit.ly/327GloL

Pareja-Eastway, M. \& Sánchez-Martínez, M. (2012). La política de vivienda en Espańa: lecciones aprendidas y retos de futuro. Revista Galega de Economía, 21(2), 203-232. https://dialnet.unirioja.es/servlet/articulo?codigo $=4246105$

Rodríguez, A. \& Sugranyes, A. (2005). El problema de vivienda de los "con techo". En A. Rodríguez \& A. Sugranyes (Eds.), Los con techo. Un desafio para la politica de vivienda social (pp. 59-78). Ediciones SUR. http://www.sitiosur.cl/r.php?id=81

Sabatini, F., Cáceres, G. \& Cerda, J. (2001). Segregación residencial en las principales ciudades chilenas: Tendencias de las tres últimas décadas y posibles cursos de acción. EURE, 28(82), 21-42. http://dx.doi.org/10.4067/S0250-71612001008200002

Scanlon, K., Whitehead, C. \& Fernández Arrigoitia, M. (2014). Introduction. En K. Scanlon, C. Whitehead \& M. Fernández Arrigoitia (Eds.), Social Housing in Europe (pp. 1-20). Wiley Blackwell.

Sugranyes, A. (2006). Social housing policy in Chile since 1980. Actors and products. Tesis doctoral. Delft University of Technology. http://resolver.tudelft.nl/uuid:9ce48f5884f4-48d1-928e-49e50f1a34b7

Torres-Tovar, C. (2008). Reflexiones sobre el hábitat y vivienda en Colombia 1990-2008. En C. M. Yory (Ed.), Pensando "en clave" de hábitat: una búsqueda por algo más que un techo (pp. 132-182). Universidad Nacional de Colombia. 